\title{
Reporte de Caso: Hernia de Amyand
}

\author{
Case Report: Hernia de Amyand
}

\section{${ }^{*}$ Jacinto Noguera Arzamendia; ${ }^{* *}$ Amanda Fretes Gomez; ${ }^{* *}$ Alejandro Caballero Rodrigues}

\author{
1. Hospital Militar Central de las FFAA, Servicio de Cirugía General. Paraguay
}

\begin{abstract}
RESUMEN
La hernia de Amyand constituye un raro tipo de herniación inguinal en la que su contenido es el apéndice vermiforme, siendo infrecuente encontrarnos con un apéndice inflamado en el interior del saco herniario, y excepcional realizar su diagnóstico preoperatorio, siendo pocos los casos descritos en la literatura. Dada la rareza del cuadro y la escasa bibliografía médica existente, resulta complicado unificar criterios sobre el manejo óptimo de esta patología. A pesar de esto, consideramos que la mejor opción es la reducción del apéndice al interior de la cavidad abdominal sin exéresis de este en los casos en los que no exista cuadro de apendicitis concomitante, junto con una reparación del defecto herniario según las técnicas habituales. Presentamos un caso de paciente de sexo masculino de 40 años de edad, sin patología de base, con diagnostico intraoperatorio de hernia de amyand, cuya resolución quirrugica fue reducción del apéndice a cavidad adbominal y correcion del defecto herniario con malla protésica.
\end{abstract}

Palabras claves: Hernia de Amyand, Apendice, Hernioplastia, Losanoff

\section{SUMMARY}

Amyand's hernia constitutes a rare type of inguinal herniation in which its content is the vermiform appendix, being uncommon to find an inflamed appendix inside the hernial sac, and exceptional to perform its preoperative diagnosis, with few cases described in the literature. Given the rarity of the condition and the scarce existing medical literature, it is difficult to unify criteria on the optimal management of this pathology. Despite this, we consider that the best option is the reduction of the appendix to the interior of the abdominal cavity without exeresis of this in cases in which there is no concomitant appendicitis, together with a repair of the hernia defect according to the usual techniques. We present a case of a 40-year-old male patient, without underlying pathology, with an intraoperative diagnosis of amyand hernia, whose surgical resolution was reduction of the appendix to the adbominal cavity and correction of the hernia defect with prosthetic mesh.

Key words: Amyand's hernia, appendix, hernioplasty, Losanoff.

\section{INTRODUCCIÓN}

La presencia del apéndice vermiforme en el interior de un saco herniario inguinal se denomina hernia de Amyand. La incidencia de hernias inguinales conteniendo el apéndice vermiforme se sitúa entorno al $0,28-1 \%{ }^{(1)}$ Normalmente se trata de una hernia inguinal que contiene el apéndice vermiforme sin inflamación, y es un hallazgo casual durante la intervención quirúrgica. Si este apéndice cecal aparece en una hernia crural, esta se denomina «hernia de Garengeot». La incidencia de una apendicitis en el saco de una hernia inguinal es del $0.07-0.13 \%{ }^{(2)}$

\section{CASO CLÍNICO}

Paciente de sexo masculino de 40 años de edad, consulta de forma ambulatoria por consultorio. En el interrogatorio refiere episodios de dolor en región inguinal derecha de larga data, desde hace 18 mes aprox. que se acompañan del esfuerzo físico, al examen físico se constata tumoración de $2 \mathrm{cms}$ de diámetro aprox. de bordes y límites netos de consistencia blanda elástica, reductible coercible en región inguinal derecha que protuye con maniobras de valsalva, no dolorosa a la palpación. Se decide programar cirugía con diagnóstico hernia inguinal indirecta no complicada.

\section{Ecografía}

Intervención terapéutica: Se realiza abordage quirúrgico según técnica de Lichtenstein, se constata saco herniario de $5 \mathrm{~cm}$ de diámetro, a la apertura del mismo se constata contenido apendice cecal no congestivo, sin signos de inflamación, por lo cual se decide la re introducción del mismo a la cavidad abdominal, se procede a la hernioplastia por la técnica anteriormente mencionada (Figura1 y 2).

\section{DISCUSIÓN}

La hernia de Amyand es una patología con muy baja prevalencia. Normalmente se trata de una hernia inguinal que contiene el apéndice vermiforme sin inflamación, y este puede encontrarse inflamado, abscesificado e incluso perforado. Se ha publicado una mortalidad, principalmente relacionada con la difusión peritoneal de la infección, de entre el $5.5 \%$ y el $30 \%{ }^{(3)}$ El diagnóstico preoperatorio de la hernia de Amyand es excep-

\footnotetext{
* Director Médico Hospital Militar Central de las FF AA

** Residente de Cirugía General - Hospital Militar Central de las FF AA

Autor correspondiente: Dr. Alejandro Caballero Rodrigues - Email: mansoncaballero87@icloud.com

Recibido: 21/05/2020 - Aceptado 10/06/2020
}

Este es un artículo publicado en acceso abierto bajo una licencia Creative Commons 


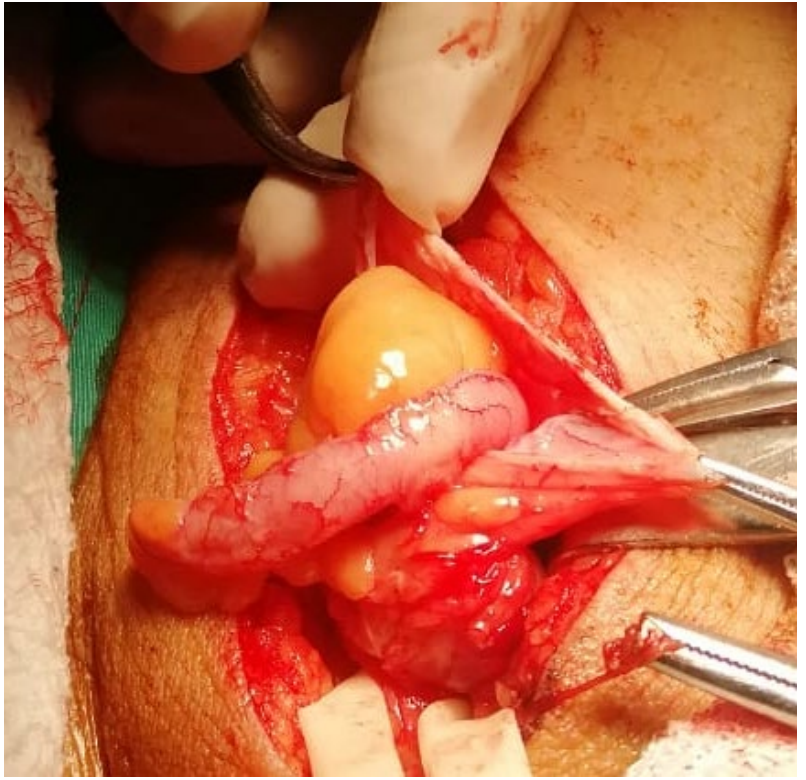

Figura 1.

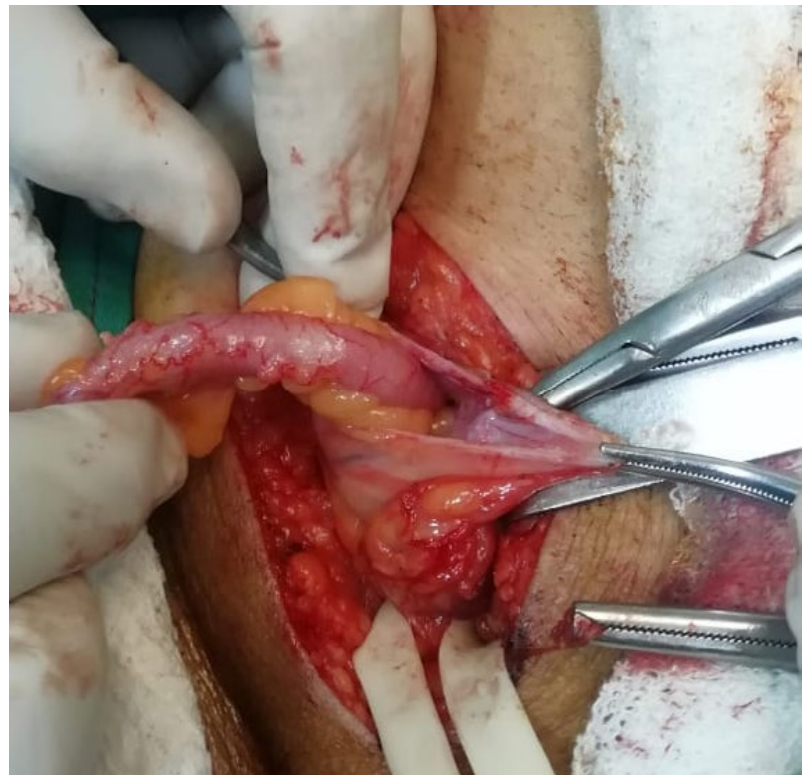

Figura 2.

\begin{tabular}{|c|c|c|c|c|}
\hline Tipo de hermia & 1 & 2 & 3 & 4 \\
\hline Apéndice & Normal & $\begin{array}{l}\text { Apendicitis aguda sin } \\
\text { peritonitis }\end{array}$ & $\begin{array}{l}\text { Apendicitis aguda con } \\
\text { peritonitis }\end{array}$ & $\begin{array}{l}\text { Apendicitis aguda asociada } \\
\text { a otra patología abdominal }\end{array}$ \\
\hline \multirow[t]{2}{*}{ Tratamiento } & $\begin{array}{l}\text { Apendicectomía } 6 \\
\text { reducción (según edad) }\end{array}$ & Apendicectomía & $\begin{array}{l}\text { Apendicectomía } \\
\text { laparotómica }\end{array}$ & $\begin{array}{l}\text { Apendicectomía } \\
\text { laparotómica }\end{array}$ \\
\hline & Hernioplastia & $\begin{array}{l}\text { Herniorrafio hernioplastia } \\
\text { con material biológica }\end{array}$ & Hemiorrafia & $\begin{array}{l}\text { Hernioplastia oherniorrafia } \\
\text { según sepsis }\end{array}$ \\
\hline
\end{tabular}

Figura 3.

cional, por lo que debe sospecharse ante una hernia inguinal derecha (excepcionalmente izquierda), incarcerada con signos de peritonitis local y datos sépticos clínico.

Analíticos sin cuadro de obstrucción intestinal ${ }^{(4)}$. Es de especial importancia, en caso de sospecha clínica, no realizar maniobras de taxis. La fiebre y la leucocitosis no son constantes, tal como se dio en nuestro caso ${ }^{(5)}$. La mayoría de las hernias de Amyand se diagnostican durante la cirugía, porque ésta se indica ante la sospecha de una hernia inguinal complicada, siendo excepcional su diagnóstico preoperatorio. La TC es muy útil para su diagnóstico en adultos, así como la ecografía lo es en niños. La hernia de Amyand ha sido definida y clasificada (clasificacion de Losanoff) en cuatro tipos según contenga un apéndice no inflamado, inflamado, perforado o un tumor apendicular o masa abdominal concomitante, proponiéndose como tratamiento la apendicectomía transherniotomía o laparotómica con hernioplastia o herniorrafia, dependiendo de la presencia de inflamación o sepsis abdominal ${ }^{(6-7)}$ (Figura 3).

\section{REFERENCIAS BIBLIOGRÁFICAS}

1. D’Alia C, Lo Schiavo MG, Tonante A, Taranto F, Gagliano E, Bonanno L, et al. Amyand's hernia: case report and review of the literature. Hernia. 2003; 7(2): 89-91.

2. Sharma H, Gupta A, Shekhawat NS, Memon B, Memon MA. Amyand's hernia: A report of 18 consecutive patiens over 15 year period. Hernia. 2007;11:31-5

3. Agirre Etxabe, L., Prieto Calvo, M., García Etxebarría, A., García González, J. Sarriugarte Lasarte, A. and Colina Alonso, A., 2020. Hernia De Amyand (Tipo
2 De Losanoff) Diagnosticada Preoperatoriamente Y Tratada Mediante Hernioplastia Con Malla Biológica.

4. Díaz Gonzaléz EE, Fleites Pérez R. Presentación de tres pacientes con hernia de Amyand. Acta Médica del Centro. 2012;6 Supl 1.

5. Fernando J, Leelaratna S. Amyand's hernia. Ceylon Med J. 2002; 47: 71.

6. Losanoff JE, Basson MD. Amyand hernia: a classification to improve management. Hernia. 2008; 12(3): 325-6. 